\title{
Opioid Taper Practices Among Clinicians
}

\author{
Amelia L Persico ${ }^{1,2}$ \\ Jeffrey J Bettinger ${ }^{2,3}$ \\ Erica L Wegrzyn ${ }^{2,4}$ \\ Jeffrey Fudin $\mathbb{D}^{2,4}$ \\ Scott A Strassels (iD)
}

'Shields Health Solutions, Stoughton, MA, 02072, USA; ${ }^{2}$ Remitigate Therapeutics, Delmar, NY, I 2054, USA; ${ }^{3}$ Saratoga Hospital Medical Group, Saratoga, NY, 12866, USA; ${ }^{4}$ Stratton VA Medical Center, Albany, NY, 12208, USA; ${ }^{5}$ The Ohio State University, Columbus, $\mathrm{OH}$, 43004, USA; ${ }^{6}$ Atrium Health, Charlotte, NC, US

Correspondence: Amelia L Persico Email apersico@remitigate.com
Introduction: Opioid dose tapers are used frequently when cross-titrating from one or more opioids to another or when discontinuing therapy. Currently, there is no universally accepted evidence-based standard of care for this procedure which can leave patients at risk for withdrawal symptoms, inadequate pain control, or elevated suicide risk.

Objective: The objective of this study was to examine practices and rationale among clinicians, to determine if there is a difference among respondents in their comfort level, method and rationale for tapering opioids at various morphine milligram equivalents (MME) and to assess the need for the development of a standard of care.

Methods: Data were derived from an electronic survey developed using SurveyMonkey ${ }^{\circledR}$. The survey was disseminated via e-mail listservs, social media, and professional organizations. Data were collected regarding profession, confidence tapering opioids at varying total MME, method and rationale for tapering, and pharmacologic management of withdrawal symptoms. Pearson's Chi squared and Fisher's exact tests were used to assess statistical significance of results.

Results: A total of 149 clinicians completed the survey, physicians, NPs, pharmacists, and PAs accounted for $51 \%, 20 \%, 19 \%$, and $10 \%$ of participants, respectively. Overall, $55 \%$ of the respondents self-identified as pain specialists. There were no statistically significant differences in reported comfort level among the different types of providers. Nearly $50 \%$ of participants indicated their rationale for tapering or discontinuing opioids was the 2016 CDC guidelines.

Conclusion: Despite that the majority of providers surveyed self-identified as pain specialists, over $50 \%$ were not comfortable tapering opioids at doses greater than $120 \mathrm{MME} /$ day. This observation suggests a need for further education and establishment of consensus guidelines on method and rationale for opioid tapering. Provider motivation for tapering was largely influenced by CDC guidelines based on low quality evidence. This strengthens the argument for the creation of guidelines based on high quality evidence.

Keywords: opioid, pain management, chronic pain

\section{Introduction}

Currently there are no consensus guidelines on how to safely and effectively taper or discontinue opioids. The available guidelines focus instead on safe and effective prescribing strategies. ${ }^{1}$ In 2016 the Center for Disease Control and Prevention (CDC) released guidelines for prescribing opioids for chronic pain. The recommendations made were largely based on case series or expert opinion. Notably per the National Guideline Clearing House, these represent type 3 or 4 evidence, yet received a GRADE A recommendation. Moreover, these guidelines exist in a backdrop where prescription opioid deaths in persons to whom they were prescribed are not delineated from illicit opioid use, nor do we know if these deaths are from a single opioid or the combination of other agents, including but 
not limited to alcohol. ${ }^{2}$ As previously published in Practical Pain Management, by far the majority of opioid-related deaths over the last several years are clearly due to illicit fentalogues. ${ }^{3}$ Unfortunately, the CDC Opioid Guidelines have had adverse, unintended consequences, in part due to their misapplication, that have in and of themselves caused increased morbidity and mortality. ${ }^{4}$ This study seeks to assess prescribers interpretation and application of at least one portion of these guidelines.

The target audience for these guidelines was primary care providers treating chronic pain patients. ${ }^{5}$ The guidelines provided recommendations to limit morphine milligram equivalents (MME) and, more specifically, to avoid increasing $>90 \mathrm{mg} /$ day without careful justification or guidance as to how to taper patients to these doses. Statements regarding MME are concerning as they were made without acknowledging the pharmacokinetic and pharmacodynamic variability among different opioids. The guidelines also failed to note the variability among MME calculations/calculators which limits standardized conversion from one opioid to another. ${ }^{5,8}$ Subsequent data has indicated that providers, payers and policy makers have misapplied these recommendations. ${ }^{8}$ For this reason we have included questions pertaining to comfort level tapering at various MME in the survey.

Prior to and in response to these guidelines several states passed laws establishing maximum daily doses for opioids and many third-party payers have followed suit by limiting coverage based on maximum MMEs. ${ }^{6,7}$ Individual providers have often erred by applying the guidelines inflexibly and without regard for patient specific factors. ${ }^{8}$ Others have misapplied the recommendations to populations beyond the scope of the guideline such as those with cancer, those receiving medication assisted treatment for opioid addiction or post-surgical patients. In a commentary published approximately three years after the writing of the guidelines two of the authors commented that better evidence is needed to guide clinical decisions regarding when and how to reduce high dose opioids for patients receiving long term therapy. ${ }^{8}$ These laws and plan limitations pose multiple problems in our health care system including limiting patient access to necessary medications, potentially putting patients in danger of opioid withdrawal if their therapy is abruptly discontinued, and increased suicidality. ${ }^{8,9}$

Our team hypothesized that in light of the CDC guidelines, lack of evidence-based recommendations for tapering opioids, and challenges translating education in health profession training to clinical practice, there would be a large amount of variability in prescriber identified expertise in tapering opioids. This variability could result in increased patient harm, morbidity and mortality. We further postulated that the results of this survey could establish a need for development of standard of care guidelines developed by experts in chronic pain management and pharmacology. The primary objective of this survey was to determine the level of variation within and between clinicians in opioid tapering methods by comparing opioid taper practices within and between respondent pharmacists, physicians, nurse practitioners and physician's assistants. The secondary objectives were to assess prescriber motivation for tapering or discontinuing opioids, determine if there is a difference among respondents in their comfort level tapering opioids at various MMEs, and to assess the need for development of a standard of care for opioid tapering.

\section{Methods}

This study was deemed exempt by the Stratton VA IRB as the study was found to pose minimal risk to the subjects. The need for informed consent was also waived under 45 CFR 46.116 and data collection was completely anonymous. This study was completed in compliance with the declaration of Helsinki. Data for this study was derived from an online survey developed using Survey Monkey ${ }^{\circledR}$ to collect information from pharmacists, physicians, nurse practitioners and physician's assistants who routinely prescribe or participate in prescribing chronic opioid therapy. The nine-question survey was disseminated via Facebook, other social media sites, and various pain management websites, including practicalpainmanagement.com. Invitations to participate were sent via e-mail as well as social media. Although participation was solicited through avenues that would be expected to attract only health care providers there was no specific criteria to ensure that only health care providers participated. Additionally, there were no specific restrictions in place to prevent individuals from taking the survey more than once. IP addresses were not collected and no personal information was gathered. Participation was completely voluntary and anonymous.

Information was collected about respondents' demographic and practice characteristics, and their comfort with opioid tapering (consider including survey in Appendix 1). A chart was provided with commonly 
prescribed opioids and MME to mitigate the shortcomings of MME calculators and eliminate variability among respondent interpretation of the questions. ${ }^{6}$ No responses were excluded from the analysis. Data were analyzed using the Pearson's chi-squared and Fisher's Exact tests.

\section{Results}

There were 149 respondents in total. Respondents' demographic and clinical practice characteristics are shown in Table 1. The survey was open from December 19, 2018 to January 29, 2019. The survey was closed when no responses were received for multiple days in a row. The average time to complete the survey was 3 minutes. The respondents were well distributed geographically with no single region of the country possessing the majority. The respondents were $51 \%$ physicians, $19 \%$ pharmacists, $20 \%$ nurse practitioners and $10 \%$ physician's assistants. A total of $82(55 \%)$ respondents self-identified as pain specialists, $11 \%$ identified as palliative care specialists, and $24 \%$ as general practice. The remaining $10 \%$ of respondents answered "none of the above" and all 149 respondents answered this question (Table 2).

Fewer than $50 \%$ of the respondents indicated that they felt comfortable tapering opioids at doses greater than 120 $\mathrm{MME} /$ day and $15 \%$ of respondents did not feel comfortable tapering opioids at any dose (Table 3 ). No statistically significant differences were noted between provider types in comfort level tapering opioids $(p=0.288)$. Relatedly, respondents were asked how they approach an opioid taper for patients on less than or equal to $60 \mathrm{MME} /$ day. Fifty (34\%) respondents answered that it depends on the patient's total daily dose and 42 (28.5\%) selected 10 $19 \%$ of total daily dose per reduction. For patients on greater than $60 \mathrm{MME} /$ day $38.5 \%$ of respondents said that they would taper the dose monthly. The next most prevalent answers were every 2 weeks and "I do not follow any specific pattern when tapering" both with $19 \%$ of respondents. There were two respondents who did not answer this question.

In assessing provider approach to opioid tapering our survey found that $50 \%$ of respondents approach a taper for a long-acting or extended release (ER) opioid by reducing the dose of the ER opioid and using a short-acting product as needed. There were 47 respondents (32\%) who would lower the dose of the ER opioid and not supply an immediate release product. Two respondents skipped this question. Providers were asked if they prescribe any pharmacological agents to blunt symptoms of withdrawal when tapering opioids. Approximately half, 74 (50\%), responded that they do not while $57(38.5 \%)$ responded that they use an alpha-2 adrenergic receptor agonist. Of note, 1 respondent skipped this question.

Table I Demographics

\begin{tabular}{|c|c|c|c|c|c|c|}
\hline Profession & $\%$ of Respondents & $\begin{array}{l}\% \text { of Respondents } \\
\text { from Northeast }\end{array}$ & $\begin{array}{l}\% \text { of Respondents } \\
\text { from Midwest }\end{array}$ & $\begin{array}{l}\% \text { of Respondents } \\
\text { from South }\end{array}$ & $\begin{array}{l}\% \text { of Respondents } \\
\text { from West }\end{array}$ & $\begin{array}{l}\text { Total } \\
\text { Respondents } \\
\text { (Raw \#) }\end{array}$ \\
\hline Physician & 51 & 24 & 15 & 34 & 27 & 76 \\
\hline Pharmacist & 19 & 35 & 19 & 31 & 15 & 28 \\
\hline Nurse Practitioner & 20 & 17 & 31 & 34 & 17 & 29 \\
\hline Physician's Assistant & 10 & 29 & 36 & 21 & 14 & 15 \\
\hline $\begin{array}{l}\text { Percent per Region (\# } \\
\text { of respondents) }\end{array}$ & - & 25 & 21 & 32 & 21 & $100(149)$ \\
\hline
\end{tabular}

Note: $P=0.0052$

Table 2 Area of Specialty

\begin{tabular}{|l|l|l|l|l|}
\hline Profession & \% General Practice & \% Pain & \% Palliative Care & \% None of the Above \\
\hline Physician & 26 & 58 & 13 & 3 \\
Pharmacist & 15 & 44 & 11 & 30 \\
Nurse Practitioner & 24 & 62 & 3 & 10 \\
Physician's Assistant & 27 & 47 & 13 & 13 \\
Percent per Specialty & 24 & 55 & 11 & 10 \\
\hline
\end{tabular}

Note: $P=0.030$. 
Table 3 Comfort Tapering at Specific Doses

\begin{tabular}{|l|l|l|l|l|l|}
\hline Profession & $\begin{array}{l}\text { I Do Not Feel } \\
\text { Comfortable } \\
\text { at Any } \\
\text { Dose (\%) }\end{array}$ & $\begin{array}{l}\text { I Am Most } \\
\text { Comfortable } \\
\text { Tapering at Doses } \\
\text { <60MME/Day (\%) }\end{array}$ & $\begin{array}{l}\text { I Am Most } \\
\text { Comfortable } \\
\text { Tapering at Doses } \\
\mathbf{6 0 - 8 9} \text { MME/Day (\%) }\end{array}$ & $\begin{array}{l}\text { I Am Most } \\
\text { Comfortable } \\
\text { Tapering at Doses } \\
\text { 90-I20 MME/ } \\
\text { Day (\%) }\end{array}$ & $\begin{array}{l}\text { I Am Most } \\
\text { Comfortable } \\
\text { Tapering at Dose } \\
\text { I I 20MME/Day (\%) }\end{array}$ \\
\hline Physician & 11 & 23 & 5 & 16 & 45 \\
Pharmacist & 7 & 26 & 7 & 26 & 33 \\
Nurse Practitioner & 23 & 19 & 4 & 7 & 27 \\
Physician's Assistant & 33 & 13 & 6 & 13 & 33 \\
Percent per Dose Range & 15 & 22 & 19 & 38 \\
\hline
\end{tabular}

Note: $P=0.288$.

The most commonly cited motivation for tapering opioids over the last year was the new CDC guidelines with $50 \%$ of respondents identifying that as one of the reasons they have tapered. The second most prevalent reason was patient misuse at $32 \%$. Patient request and insurance regulations tied for third most commonly identified reason for tapering opioids with $29 \%$ of respondents citing each as one of the reasons they have tapered opioids in the last year (Figure 1). Of note, 4 respondents skipped this question.

\section{Discussion}

This survey of 149 clinician respondents highlights the discomfort many providers have in tapering opioid therapy and the wide variability in both method and rationale for tapering. The findings bolster the authors' hypothesis that due to a lack of evidence-based recommendations for tapering opioids there would be a large amount of variability in prescriber identified expertise in tapering opioids. These findings are further supported by the multitude of variability in available literature regarding opioid tapers. Of the limited data and guidance available regarding safe and effective tapering of opioids, Pergolizzi et al suggest a patient specific approach emphasizing shared decision making and a 5-20\% decrease in dose every 4 weeks. ${ }^{10}$ The process can take months or years depending on how long a patient has been on opioids, their total daily dose and other various patient specific factors. ${ }^{7}$ While prior literature released by the $\mathrm{CDC}$ and Washington State Agency Medical Directors' Group advocate a reduction of $10 \%$ per week while, yet another guideline recommends decreasing total daily opioid dose by $20 \%$ per week if the patient is high risk. ${ }^{11}$

Dr. Jennifer Schneider wrote, in Practical Pain Management, that the addition of clonidine to a taper protocol can allow for a faster taper and that patients



Figure I A summary of provider responses to the following question: In the past year, if you have tapered opioids, what was the most common reason? (check all that apply). The choices are displayed on the $\mathrm{Y}$-axis and the percent of respondents selecting each option is displayed on the $\mathrm{X}$-axis. 
who are being tapered because their pain has resolved (ie hip or knee replacement) can tolerate a faster taper than those with chronic pain. ${ }^{12}$ Notably, our results showed that few providers utilized pharmacotherapy to blunt the symptoms of withdrawal, despite this being one of few recommendations identified in the literature for safe opioid tapering. Proposed taper protocols often emphasize that the longer a patient has been on opioid therapy the longer the interval between dose reductions should be. ${ }^{1,10-13}$ A 2019 qualitative study of patients taking opioids identified three overarching themes that have impacted patient's lives when they are faced with an opioid taper - deepening of stigma, loss of autonomy, and an increase in preexisting structural vulnerabilities among patients who take opioids. ${ }^{13}$ These represent unintended consequences of the misapplication of currently available guidance and further reinforce the authors' hypothesis that increased morbidity and mortality could result from the lack of highquality evidence based guidelines available. What is apparent from a review of the literature coupled with the results of this, albeit small, study is that there is a multitude of approaches to tapering patients off of opioid therapy. Vast interindividual variability among patients and providers alike reminds us that patient centered care should remain at the core of all practices. It provides further support for an individualized approach to opioid tapering. ${ }^{11}$ In sum, the array of different resources available and lack of one reliable consensus guideline regarding opioid taper recommendations is likely responsible for the vast variability among provider tapering practices.

Despite the fact that the majority of providers surveyed self-identified as pain specialists, $15 \%$ reported that they were not comfortable tapering opioids at all. This fact alone, juxtaposed to the reality that providers are tapering patients off of their opioids anyway, is alarming and provides rationale for development of expert consensus guidelines for opioid tapering. As we found that motivation for tapering opioids was strongly influenced by CDC guidelines and insurance regulations rather than medical reasons or patient specific factors it could be valuable to emphasize a return to evidence based, patient-centered medicine in pain management. We posit that these providers are uncomfortable tapering opioids due to a lack of evidencebased consensus guidelines.

Our data demonstrate that provider comfort level with tapering opioids corresponded with something more than just starting dose or daily MMEs. The field would benefit from further research to establish what factors contribute to provider comfort level tapering opioids. We would also like to see research exploring what factors limited prescribing of pharmacological agents to blunt symptoms of withdrawal when tapering or discontinuing opioids. Examination of a group of providers who do not selfidentify as pain specialists could also be enlightening, particularly considering the intended audience for the 2016 CDC guidelines was purported to be primary care providers.

There are multiple inherent limitations in a survey and our study was not invulnerable to these pitfalls. In addition to those inherent weaknesses, our survey has information bias as respondents were permitted to skip questions, therefore each question did not necessarily have a $100 \%$ response rate. There was also no mechanism to prevent respondents from participating in the survey more than once as there was no restriction on number of responses per individual or IP address since this information was not tracked in order to maintain anonymity. There is also selection bias as only those who received the link via listservs, email or involvement in specific professional organizations could participate. As the survey included multiple choice questions it did not account for every possible response thus there is the risk that incomplete information was collected. Opportunity for qualitative responses could have enriched the data collected and represents an opportunity for further study. Further, the results of this survey are mostly applicable to pain specialists as the majority of our respondents identified themselves as such. A larger, more diverse population would have yielded more generalizable results across multiple specialties. That being said, the data are generalizable across multiple professions as there were no statistically significant differences among the different respondent professions. Finally, the data collected were all based on selfreport. Our hope is that the anonymity inspired honest responses, however, this cannot be guaranteed and thus represents a potential weakness.

\section{Conclusion}

Given the dearth of high-quality research, the 2016 CDC guidelines encouraged providers to reduce patient access to opioids without regard for the quality of the evidence or safe and effective opioid tapering practices. The data gathered in this survey suggest that providers require more direction and guidance regarding how to safely and appropriately taper opioid doses for patients who may benefit. The survey results showing that many clinicians 
are uncomfortable tapering opioids and that there is variability among opioid taper practices demonstrate the need for consensus guidelines on safe and effective opioid taper techniques. Ideally, these guidelines would be formulated by an interdisciplinary panel of chronic pain management experts and would urge intelligent, individualized application to provide patient-centered care to some of the most at-risk populations.

\section{Disclosure}

Dr Jeffrey $\mathrm{J}$ Bettinger reports personal fees for advisory board from Hisamitsu Pharmaceutical Co., Inc, outside the submitted work. Dr Erica L Wegrzyn reports personal fees for consultancy from Remitigate LLC, outside the submitted work. Dr Jeffrey Fudin provided lectures (nonspeaker bureau) for Abbott Laboratories, speakers bureau, consulting, publications for AcelRx Pharmaceuticals, collaborative publications, consulting, advisory boards for BioDelivery Sciences International, micro serum testing for substances of abuse (consulting) for Firstox Laboratories, advisory boards, collaborative non-paid poster presentations for GSK, advisory board for Hisamitsu America Inc, advisory board for Hikma Pharmaceuticals, collaborative non-paid publications for Scilex, speakers bureau, consultant, advisory boards for Salix, advisory board for Kempharm, lecture (non speakers bureau) for Torrent Pharmaceuticals, outside the submitted work. The authors report no other conflicts of interest in this work.

\section{References}

1. Chantal B, Kulich R, Rathmell J. Tapering long-term opioid therapy in chronic noncancer pain: evidence and recommendations for everyday practice. Mayo Clin Proc. 2015;90(6):828-842.
2. Kertesz SG. Turning the tide or riptide? The changing opioid epidemic. Subst Abus. 2017;38(1):3-8. doi:10.1080/08897077.2016. 1261070

3. Persico AL, Wegrzyn EL, Fudin J, Schatman ME. Fentalogues. J Pain Res. 2020;13:2131. doi:10.2147/JPR.S265901

4. Darnall BD, Juurlink D, Kerns RD, et al. International stakeholder community of pain experts and leaders call for an urgent action on forced opioid tapering. Pain Med. 2019;20(3):429-433. doi:10.1093/ $\mathrm{pm} / \mathrm{pny} 228$

5. Dowell D. Haegerich CDC guideline for prescribing opioids for chronic pain- United States, 2016. Recomm Rep. 2016;65(1):1-49.

6. Rennick A, Atkinson T, Cimino NM, et al. Variability in opioid equivalence calculations. Pain Med. 2015. doi:10.1111/pme.12920

7. Bulloch M. Opioid prescribing limits across the states; February 05, 2019. Available From: https://www.pharmacytimes.com/contributor/ marilyn-bulloch-pharmd-bcps/2019/02/opioid-prescribing-limitsacross-the-states. Accessed October 4, 2021.

8. Dowell D, Haegerich T, Chou R. No shortcuts to safer opioid prescribing. NEJM. 2019;380(24):2285-2287. doi:10.1056/NEJMp 1904190.

9. Oliva EM, Bowe T, Manhapra A, et al. Associations between stopping prescriptions for opioids, length of opioid treatment and overdose or suicide deaths in US veterans: an observational evaluation. BMJ. 2020;368:m283. doi:10.1136/bmj.m283

10. Pergolizzi JV, Rosenblatt M, Mariano DJ, Bisney J. Tapering opioid therapy: clinical strategies. Pain Manag. 2018;8(6):409-413. doi:10. 2217/pmt-2018-0040

11. Kominek C. What's the safest, effective way to taper a patient off of opioid therapy. PPM. 2018;18(5). Available from: https://www.practi calpainmanagement.com/treatments/pharmacological/tapering/whatsafest-effective-way-taper-patient-opioid-therapy. October 15, 2021.

12. Schneider J. Tapering a patient off opioids. PPM. (Originally published in 2012 and updated in 2019;12(2). Available from: https:// www.practicalpainmanagement.com/treatments/pharmacological/ tapering/tapering-patient-opioids. Accessed Octoer 15, 2021.

13. Antoniou T, Ala-Leppilampi K, Shearer D, et al. "Like being put on an ice floe and shoved away": a qualitative study of the impacts of opioid-related policy changes on people who take opioids. Int J Drug Policy. 2019;66:15-22. doi:10.1016/j.drugpo.2019.01.015
Journal of Pain Research

\section{Publish your work in this journal}

The Journal of Pain Research is an international, peer reviewed, open access, online journal that welcomes laboratory and clinical findings in the fields of pain research and the prevention and management of pain. Original research, reviews, symposium reports, hypothesis formation and commentaries are all considered for publication. The manuscript management system is completely online and includes a very quick and fair peer-review system, which is all easy to use. Visit http:// www.dovepress.com/testimonials.php to read real quotes from published authors. 